\title{
First Draft Genome Sequence Resource of Colletotrichum liriopes Causing Leaf Anthracnose on Ophiopogon japonicus
}

\author{
Mingshuang Wang, ${ }^{1,2, \dagger}$ Xiujun Luo, ${ }^{1,2}$ and Huizhong Wang ${ }^{1,2, \dagger}$ \\ ${ }^{1}$ College of Life and Environmental Sciences, Hangzhou Normal University, Hangzhou, 310036, China \\ ${ }^{2}$ Zhejiang Provincial Key Laboratory for Genetic Improvement and Quality Control of Medicinal Plants, \\ Hangzhou Normal University, Hangzhou, 310036, China
}

\begin{abstract}
Leaf anthracnose of Ophiopogon japonicus is an important disease that can significantly reduce the quality and economic value of this traditional Chinese medicinal plant. The disease is caused by Colletotrichum liriopes, a necrotrophic fungus that belongs to the Glomerellaceae family of the Sordariomycetes class. Here, we present the draft whole-genome sequence of the $C$. liriopes strain A2 that caused leaf anthracnose on $O$. japonicus. The assembly consists of 407 contigs with an estimated genome size of $53.1 \mathrm{Mb}$. Furthermore, we identified 670 carbohydrate-active enzymes, 1,377 secreted proteins, and 60 secondary metabolite gene clusters, which may be associated with the pathogenicity of this pathogen. This genome resource will provide a valuable resource for future research on the pathogenesis of $C$. liriopes and comparative genome analyses within genus Colletotrichum.
\end{abstract}

Ophiopogon japonicus (Thunb.) Ker-Gawl (Liliaceae) is a traditional Chinese medicinal plant widely cultured in China. It is evergreen, perennial, tufted, and grass-like. The leaves are linear and 20 and $40 \mathrm{~cm}$ long. Ophiopogonis Radix, the root of $O$. japonicus, admitted as one of the functional food ingredients by the Ministry of Health of the People's Republic of China, is used as the main ingredient in many prescriptions. According to the traditional Chinese medicine principle, Ophiopogonis Radix nourishes the yin, promotes body fluid production, moistens the lungs, eases the mind, and clears away heart fire (Liang et al. 2012). Over thousands of years, $O$. japonicus has been used to relieve symptoms such as phlegm, coughing, and heat in the lungs caused by bacterial infections and to alleviate diabetes mellitus and constipation (Chen et al. 2016). However, leaf anthracnose caused by Colletotrichum liriopes has become an important disease during $O$. japonicus cultivation in recent years (Wang and Wang 2021). At the early stage of infection, one or several small patches with brown edges and yellow halos are formed at the middle part of the leaf; subsequently, the lesions enlarged to irregular shapes; finally, patches keep proceeding outward and form a blight (Wang and Wang 2021). The disease is very severe in years with high temperatures and heavy rainfall, which seriously affects the yield and quality of $O$. japonicus (Wang and Wang 2021).

The causal agent of this important leaf disease was identified as $C$. liriopes, which belongs to the Glomerellaceae family of the Sordariomycetes class (Wang and Wang 2021). Thus far, studies on the mechanisms underlying the ability of $C$. liriopes to effectively infect $O$. japonicus leaves and its interaction with host cells are limited due to its recent discovery. The genes involved in nutrient absorption, vegetative growth, sporulation, stress response, and secondary metabolite biosynthesis of this pathogen remain to be identified. For these reasons, we have fully sequenced the genome of strain $\mathrm{A} 2$ of $C$. liriopes that caused leaf anthracnose on $O$. japonicus.

${ }^{\dagger}$ Corresponding authors: M. Wang; mswang@hznu.edu.cn; and H. Wang; whz62@163.com

The author(s) declare no conflict of interest.

Accepted for publication 27 November 2020
Funding

This work was supported by the Major Increase or Decrease Program in the Central Finance Level (2060302) and Zhejiang Provincial Key Research \& Development Project Grants (2018C02030).

\section{Keywords}

Colletotrichum liriopes, genome, leaf anthracnose, Ophiopogon japonicus, pathogenesis 
Table 1. Genome statistics of Colletotrichum liriopes strain A2 (accession number JADCKO000000000)

\begin{tabular}{lc} 
Features & Strain A2 \\
Genome size (Mb) & 53.1 \\
BUSCOs (\%) & 99.7 \\
Number of contigs & 407 \\
$N_{50}(\mathrm{~kb})$ & 288 \\
GC content (\%) & 52.7 \\
Protein-coding genes & 13,077 \\
Gene density (number of genes per Mb) & 246 \\
Average intron number & 2.2 \\
Average intron length & 95 \\
Average exon number & 3.2 \\
Average exon length & 440 \\
Mean gene length (bp) & 1,625 \\
Number of genes with intron & 10,553 \\
tRNA genes & 385 \\
Repeat rate (\%) & 1.98 \\
Carbohydrate-active enzymes & 670 \\
Secreted proteins & 1,377 \\
Small secreted cysteine-rich proteins & 269 \\
Secondary metabolite gene clusters & 60 \\
\hline
\end{tabular}

Genome sequencing and assembly. The reference $C$. liriopes strain, A2, was isolated from an infected $O$. japonicus leaf from Zhejiang, China (Wang and Wang 2021). This strain was ascertained as the causal agent of the leaf anthracnose of $O$. japonicus using Koch's postulates previously in the first report (Wang and Wang 2021) and was stored in 20\% glycerol solutions at $-80^{\circ} \mathrm{C}$ until use. Fungal colonies were grown on potato dextrose agar at $25^{\circ} \mathrm{C}$ and mycelia were obtained by growing spores in liquid potato dextrose broth (PDB) incubated on a rotary shaker at $160 \mathrm{rpm}$ at $25^{\circ} \mathrm{C}$ for 3 days. The genomic DNA of $C$. liriopes strain A2 was extracted from the mycelia with the cetyltrimethylammonium bromide method (Möller et al. 1992). A short insert library (350 bp) was constructed and sequenced on an Illumina HiSeq 4000 platform (Nanjing Personalbio Co., Ltd), generating 150-bp paired-end reads. Raw reads were trimmed of adapter sequences and low-quality reads using Trimmomatic v. 0.38 (Bolger et al. 2014). In total, $4.0 \mathrm{~Gb}$ of clean reads was generated in the sequencing process, which corresponds to approximately 75 -fold of sequence depth. The genome was de novo assembled into 407 contigs ( $>1 \mathrm{~kb}$ ) using SPAdes ver. 3.14.1 (Bankevich et al. 2012) and resulted in a genome size of $53.1 \mathrm{Mb}$ with an $\mathrm{N}_{50}$ of $288 \mathrm{~kb}$ (Table 1). The completeness of the genome assembly was estimated by BUSCO v4.0.2 based on lineagespecific profile library sordariomycetes_odb10 (Simão et al. 2015). In total, 3,807 of 3,817 (99.7\%) BUSCO groups in the $C$. liriopes strain A2 genome (Table 1) were identified, indicating a very high degree of completeness.

The overall $\mathrm{G}+\mathrm{C}$ content of the $C$. liriopes strain $A 2$ assembly was $52.7 \%$. The tRNA and its secondary structure were predicted by tRNAscan-SE ver. 2.0 (Chan and Lowe 2019), revealing 385 tRNA genes (Table 1). Simple repeats, transposable elements, low-complexity sequences, and satellites were detected and annotated using RepeatMasker open 4.1.0 (http://www.repeatmasker.org). Results showed that repetitive sequences represented $1.98 \%$ of the genome (Table 1). The actual repeat content is expected to be larger because small contigs shorter than $1 \mathrm{~kb}$ were filtered out, given that they may contain many repetitive sequences.

Genome annotation. To obtain high-quality gene calls, RNA sequencing (RNA-seq) was performed with the same sample (mycelia harvested from PDB) and $6.0 \mathrm{~Gb}$ of Illumina paired-end reads was obtained. RNA-seq data were then aligned to the genome using HISAT ver. 2.0.9 (Kim et al. 2019). Genome annotation was performed using the BRAKER version 2.0 pipeline (Hoff et al. 2019) based on GeneMark-ES ver. 4.48 (Borodovsky and Lomsadze 2011) and Augustus ver. 2.5.5 (Stanke et al. 2008), combining the RNA-seq-based gene prediction and ab initio gene prediction. In total, 13,077 protein-coding genes were predicted in the $C$. liriopes strain A2 genome, with an average gene length of 1,625 bp (Table 1). To functionally annotate the 13,077 genes in the $C$. liriopes strain A2 genome, we classified their protein products into protein families using the Pfam ver. 32.0 databases (El-Gebali et al. 2018). Results showed that 9,355 genes contained Pfam protein domains, and they were assigned to 3,918 protein families. 
Carbohydrate-active enzymes (CAZymes) build and breakdown complex carbohydrates and glycoconjugates and many of them play crucial roles during fungal infection (Kubicek et al. 2014). CAZymes were annotated by the web-based dbCAN2 meta server (supported by $\geq 2$ tools) (Zhang et al. 2018). In total, 670 putative CAZyme genes were identified in C. liriopes strain A2, which included 344 glycoside hydrolases, 101 glycosyl transferases, 125 auxiliary activities, 49 carbohydrate esterases, 41 polysaccharide lyases, and 10 carbohydrate-binding modules.

Fungal pathogens can secrete a number of proteins that are deployed to the hostpathogen interface during infection, to help overcome the host defense responses and acquire nutrients (Lo Presti et al. 2015). Putative extracellular proteins were identified as secreted proteins if they contain a signal peptide, located at the extracellular subcellular, and lack transmembrane domains and glycosylphosphatidylinositol (GPI)-anchor signals. To identify secreted proteins, we use SignalP 5.0 (Armenteros et al. 2019) and TMHMM ver. 2.0 (Krogh et al. 2001) to predict the signal peptide and transmembrane domains (PredHel $<2$ ) and we excluded nonextracellular and GPI-anchored proteins by using TargetP 1.1 (Emanuelsson et al. 2000) and PredGPI (Pierleoni et al. 2008). Approximately $10.5 \%$ of the total proteins $(1,377)$ of $C$. liriopes strain A2 were predicted to be secreted. The gene ontology (GO) enrichment in molecular functions of the secreted proteins was then produced with the dcGO database (Fang and Gough 2013). Hydrolase activity was the most abundant molecular function of the extracellular proteins. Other GO terms overrepresented among the secreted proteins include carboxylic ester hydrolase activity, exopeptidase activity, serine hydrolase activity, lipase activity, oxidoreductase activity, and transferase activity. All resulting secreted proteins that were shorter than 200 amino acids in length and contained at least four cysteine residues were considered to be small secreted cysteine-rich proteins (SSCPs) (Wang et al. 2019). According to this standard, 269 of them were SSCPs. Only 41 SSCPs contained functional domains and the functions of other SSCPs were largely unknown.

Secondary metabolites, especially fungal toxins, are believed to be involved in the pathogenicity of many plant-pathogenic fungal species and can be described as potential virulence factors. Fungal secondary metabolite pathways were predicted using the online tool antiSMASH 4.0 (Blin et al. 2017). In total, 60 secondary metabolite biosynthesis clusters were identified in the $C$. liriopes strain A2. These clusters comprised 22 polyketide synthase (PKS) clusters, 14 nonribosomal peptide synthetase (NRPS) clusters, 9 terpene clusters, 7 indole clusters, 4 PKS-NRPS clusters, 3 NRPS- $\beta$-lactone clusters, and 1 PKS-indole cluster. These clusters contain backbone genes of some known clusters such as the dimethylcoprogen cluster from Alternaria alternata (BGC0001249), ACT-Toxin II cluster from A. alternata (BGC0001254), naphthopyrone cluster from Aspergillus nidulans (BGC0000107), pyranonigrin E from A. niger (BGC0001124), fusarin cluster from Fusarium verticillioides (BGC0000064), and alternapyrone cluster from Alternaria solani (BGC0000012); however, further studies are requested to confirm whether $C$. liriopes is able to synthesize these metabolites.

In the present study, we provide the first-draft genome sequence of $C$. liriopes, a fungal pathogen causing leaf anthracnose on the traditional Chinese medicinal plant $O$. japonicus. The sequence represents a useful resource for future research on understanding the underlying virulence mechanisms of this economically important fungal pathogen.

Data availability. The Whole-Genome Shotgun project of $C$. liriopes strain A2 has been deposited at DNA Data Bank of Japan/European Nucleotide Archive/GenBank under accession number JADCKO000000000 (BioProject PRJNA669110 and BioSample SAMN16434550). The version described here is version JACBJF010000000. The data were set to be automatically released on 15 November 2020 .

\section{Literature Cited}

Armenteros, J. J. A., Tsirigos, K. D., Sønderby, C. K., Petersen, T. N., Winther, O., Brunak, S., von Heijne, G., and Nielsen, H. 2019. SignalP 5.0 improves signal peptide predictions using deep neural networks. Nat. Biotechnol. 37:420-423.

Bankevich, A., Nurk, S., Antipov, D., Gurevich, A. A., Dvorkin, M., Kulikov, A. S., Lesin, V. M., Nikolenko, S. I., Pham, S., and Pribelski, A. D. 2012. SPAdes: A new genome assembly algorithm and its applications to single-cell sequencing. J. Comput. Biol. 19:455-477.
Blin, K., Wolf, T., Chevrette, M. G., Lu, X., Schwalen, C. J., Kautsar, S. A., Suarez Duran, H. G., de Los Santos, E. L., Kim, H. U., and Nave, M. 2017. antiSMASH 4.0-Improvements in chemistry prediction and gene cluster boundary identification. Nucleic Acids Res. 45:W36-W41.

Bolger, A. M., Lohse, M., and Usadel, B. 2014. Trimmomatic: A flexible trimmer for Illumina sequence data. Bioinformatics 30:2114-2120.

Borodovsky, M., and Lomsadze, A. 2011. Eukaryotic gene prediction using GeneMark.hmm-E and GeneMark-ES. Curr. Protoc. Bioinf. 35:4.6.1-4.6.10. 
Chan, P. P., and Lowe, T. M. 2019. tRNAscan-SE: Searching for tRNA genes in genomic sequences. Pages 1-14 in: Gene Prediction. M. Kollmar, ed. Methods in Molecular Biology, Vol. 1962. Humana, New York, NY, U.S.A.

Chen, M.-H., Chen, X.-J., Wang, M., Lin, L.-G., and Wang, Y.-T. 2016. Ophiopogon japonicus-A phytochemical, ethnomedicinal and pharmacological review. J. Ethnopharmacol. 181:193-213.

El-Gebali, S., Mistry, J., Bateman, A., Eddy, S. R., Luciani, A., Potter, S. C., Qureshi, M., Richardson, L. J., Salazar, G. A., and Smart, A. 2018. The Pfam protein families database in 2019. Nucleic Acids Res. 47:D427-D432.

Emanuelsson, O., Nielsen, H., Brunak, S., and Von Heijne, G. 2000. Predicting subcellular localization of proteins based on their N-terminal amino acid sequence. J. Mol. Biol. 300:1005-1016.

Fang, H., and Gough, J. 2013. DcGO: Database of domain-centric ontologies on functions, phenotypes, diseases and more. Nucleic Acids Res. 41: D536-D544.

Hoff, K. J., Lomsadze, A., Borodovsky, M., and Stanke, M. 2019. Whole-genome annotation with BRAKER. Pages 65-95 in: Gene Prediction. M. Kollmar, ed. Methods in Molecular Biology, Vol. 1962. Humana, New York, NY, U.S.A.

Kim, D., Paggi, J. M., Park, C., Bennett, C., and Salzberg, S. L. 2019. Graph-based genome alignment and genotyping with HISAT2 and HISAT-genotype. Nat. Biotechnol. 37:907-915.

Krogh, A., Larsson, B., von Heijne, G., and Sonnhammer, E. L. 2001. Predicting transmembrane protein topology with a hidden Markov model: Application to complete genomes. J. Mol. Biol. 305:567-580.

Kubicek, C. P., Starr, T. L., and Glass, N. L. 2014. Plant cell wall-degrading enzymes and their secretion in plant-pathogenic fungi. Annu. Rev. Phytopathol. 52:427-451.
Liang, H., Xing, Y., Chen, J., Zhang, D., Guo, S., and Wang, C. 2012. Antimicrobial activities of endophytic fungi isolated from Ophiopogon japonicus (Liliaceae). BMC Complement. Altern. Med. 12:238.

Lo Presti, L., Lanver, D., Schweizer, G., Tanaka, S., Liang, L., Tollot, M., Zuccaro, A., Reissmann, S., and Kahmann, R. 2015. Fungal effectors and plant susceptibility. Annu. Rev. Plant Biol. 66:513-545.

Möller, E. M., Bahnweg, G., Sandermann, H., and Geiger, H. H. 1992. A simple and efficient protocol for isolation of high molecular weight DNA from filamentous fungi, fruit bodies, and infected plant tissues. Nucleic Acids Res. 20: 6115-6116.

Pierleoni, A., Martelli, P. L., and Casadio, R. 2008. PredGPI: A GPI-anchor predictor. BMC Bioinf. 9:392.

Simão, F. A., Waterhouse, R. M., loannidis, P., Kriventseva, E. V., and Zdobnov, E. M. 2015. BUSCO: Assessing genome assembly and annotation completeness with single-copy orthologs. Bioinformatics 31:3210-3212.

Stanke, M., Diekhans, M., Baertsch, R., and Haussler, D. 2008. Using native and syntenically mapped CDNA alignments to improve de novo gene finding. Bioinformatics 24:637-644.

Wang, M., Fu, H., Shen, X. X., Ruan, R., Rokas, A., and Li, H. 2019. Genomic features and evolution of the conditionally dispensable chromosome in the tangerine pathotype of Alternaria alternata. Mol. Plant Pathol. 20:1425-1438.

Wang, M., and Wang, H. 2021. First report of leaf anthracnose caused by Colletotrichum liriopes on Ophiopogon japonicus in China. Crop Prot. 140:105418.

Zhang, H., Yohe, T., Huang, L., Entwistle, S., Wu, P., Yang, Z., Busk, P. K., Xu, Y., and Yin, Y. 2018. dbCAN2: A meta server for automated carbohydrate-active enzyme annotation. Nucleic Acids Res. 46:W95-W101. 